\title{
Escala de Motivação para a Leitura para Estudantes do Ensino Fundamental: Construção e Validação
}

\author{
Reading Motivation Scale for Elementary Education Students: \\ Construction and Validation
}

\author{
Maria Aparecida Mezzalira Gomes*, a \& Evely Boruchovitch ${ }^{b}$ \\ ${ }^{a}$ Escola Superior de Educação Física de Jundiai, Jundiai, SP, Brasil \\ $\&{ }^{b}$ Universidade Estadual de Campinas, Campinas, SP, Brasil
}

\begin{abstract}
Resumo
O objetivo deste estudo é descrever os passos relativos à construção e validação de uma escala para avaliar a motivação em leitura e apresentar a análise preliminar de suas propriedades psicométricas. Participaram 253 estudantes brasileiros de $3^{\circ}$ ao $5^{\circ}$ anos de escolas públicas do Ensino Fundamental. Pela análise fatorial exploratória, emergiram quatro fatores: Motivação Extrínseca Autônoma para a Leitura, Desmotivação para a Leitura, Motivação Extrínseca Controlada para a Leitura e Motivação Intrínseca para a Leitura, que explicaram $42,71 \%$ da variância total. A consistência interna da escala total, aferida pelo Alpha de Cronbach, foi de 0,83 e dos quatro fatores variaram de 0,80 a 0,87 . Acredita-se que a escala apresenta evidências de validade e confiabilidade para utilização no contexto escolar.

Palavras-chave: Avaliação psicoeducacional, construção de instrumento, Escala de Motivação para a Leitura, leitura, Teoria da Autodeterminação.
\end{abstract}

\begin{abstract}
The purpose of this study was to describe the steps of constructing and validating a reading motivation scale for elementary education students as well as to present a preliminary analysis of its psychometric properties. The sample was composed of 253 Brazilian students, from $3^{\text {rd }}$ to $5^{\text {th }}$ grades, of Primary Education Public Schools. The exploratory factorial analyses identified four factors: Extrinsic Autonomous Motivation for Reading, Demotivation for Reading, Extrinsic Controlled Motivation for Reading and Intrinsic Motivation for Reading which explained $42.71 \%$ of the total variance. The internal consistency of the scale, estimated by Cronbach alpha, was .83 and ranged from .80 to .87 for the four factors. The scale has evidence of validity and reliability for its use in school context. Keywords: Psychoeducational evaluation, instrument construction, Reading Motivation Scale, reading, Self-determination Theory.
\end{abstract}

Diversos estudos nacionais e estrangeiros mostram que estudantes motivados para aprender conseguem melhores resultados na escola (Beker, McElvany, \& Kortenbruck, 2010; Boruchovitch, 2008a, 2008b; Guay, Marsh, Dowson, \& Larose, 2005; Joly \& Prates, 2011; Martinelli \& Genari, 2009; Prates, Joly, Dias, \& Almeida, 2011; Ratelle, Guay, Vallerand, Larose, \& Senécal, 2007; Zenorini, Santos, \& Monteiro, 2011). Nessa mesma direção, Clark e De Zoysa (2011); Taboada, Tonks, Wigfield e Guthrie (2009) investigaram e encontraram relações positivas entre a motivação do estudante como leitor e o seu sucesso

\footnotetext{
* Endereço para correspondência: Rua Rodrigo Soares de Oliveira, s/n, Anhangabau, Jundiaí, SP, Brasil 13208120. E-mail: cidgom@uol.com.br e evely@unicamp.br As autoras agradecem o apoio do Conselho Nacional de Desenvolvimento Científico e Tecnológico (CNPq) a este projeto de pesquisa realizado como parte do Pós-doutorado Júnior da primeira autora, sob a coordenação da segunda autora.
}

escolar. No relatório do Programme for International Student Assessment (PISA), a motivação para a leitura, assim como o tempo despendido nesta atividade foram considerados variáveis importantes no desempenho dos participantes dessa avaliação internacional. No entanto, houve evidência do decréscimo da motivação pela leitura quando foram comparados dados das avaliações de $2000 \mathrm{e}$ 2009 e o mesmo ocorreu nas médias obtidas por diversos países conforme se pode verificar nas publicações editadas pela Organisation de Coopération et de Développement Économiques (OCDE, 2011). Muitos pesquisadores de diferentes nacionalidades buscaram compreender as raízes desses problemas examinando os processos de alfabetização e de desenvolvimento da leitura (Manata, 2011; Mata, Monteiro, \& Peixoto, 2009; Oliveira, Boruchovitch, \& Santos, 2008) e propondo, inclusive, programas instrucionais com o objetivo de promover a motivação para ler e a compreensão leitora, visto que sua repercussão favorável na vida escolar dos estudantes é consenso entre estudio- 
Gomes, M. A. M. \& Boruchovitch, E. (2015). Escala de Motivação para a Leitura para Estudantes do Ensino Fundamental: Construção e Validação.

sos do assunto (Gomes \& Boruchovitch, 2011; Guthrie, McRae, \& Klauda, 2007).

Um aspecto importante a ser considerado é que a realização de pesquisas que lidem com diferentes variáveis educacionais exige instrumentos confiáveis, válidos e precisos. Investigações de pesquisadores americanos ligados à Psicologia Sociocognitiva, particularmente à Teoria da Autodeterminação (TAD) trouxeram contribuições valiosas no sentido de elaborar e validar escalas de avaliação da motivação do aluno para aprender. Esses instrumentos, por sua vez, têm se mostrado muito úteis para esclarecer as relações entre diferentes variáveis educacionais. Entre outros estudos, podem ser mencionados Gillet, Vallerand, e Lafrenière (2012), que pesquisaram formas de suporte à autonomia e seus efeitos na motivação de estudantes dos nove aos 17 anos. Corpus e Wormington (2011), e Wormington, Corpus e Anderson (2011) estudaram aspectos qualitativos da motivação para o estudo e suas relações com o desempenho e o envolvimento nas tarefas escolares.

A literatura nacional tem crescido, principalmente na última década, em todos os segmentos da escolarização formal. Assim, Neves e Boruchovitch (2007) e Rufini, Bzuneck e Oliveira (2011), desenvolveram estudos para validar uma escala de motivação para estudantes do Ensino fundamental. Megliato (2011) estudou perfis motivacionais de estudantes do Ensino Fundamental, para identificar as possíveis relações entre a motivação para matemática e a utilização de estratégias metacognitivas, como indícios do seu envolvimento no estudo. Zenorini e Santos (2010) construíram e validaram uma escala de motivação destinada a avaliar as metas de realização como medida da motivação escolar. Os estudos de Boruchovitch (2008a, 2008b), Joly e Prates (2011) e Rosecler e Guimarães (2010) tiveram como objetivo a avaliação da motivação de estudantes universitários. Entretanto, não foram encontrados estudos brasileiros relacionados à motivação para ler e à sua avaliação.

Nas duas últimas décadas foram disponibilizadas, nos Estados Unidos, diversas escalas para avaliar a motivação para a leitura, que, submetidas a análises psicométricas, demonstraram níveis satisfatórios em termos de validade, precisão e confiabilidade. Muitas delas têm sido validadas e utilizadas em diversos outros países. As principais dimensões abrangidas por esses instrumentos se referem às atitudes (McKenna, Kear, \& Ellsworth, 1995; à percepção de autoeficácia (Melnick, Henk, \& Marinak, 2009; Monteiro, Mata, \& Peixoto, 2008); ao autoconceito como leitor e ao valor atribuído à leitura (Gambrell, Palmer, Codling, \& Mazzoni, 1996). Um instrumento que inclui diversas variáveis relacionadas à motivação para a leitura, tais como a Importância (o Valor) e a Curiosidade, a Motivação Intrínseca e Extrínseca, o Reconhecimento Social, as Razões Sociais, o Prazer e a Autopercepção de Competência, numa perspectiva de compromisso do leitor e motivação para a realização foi elaborado e validado por Baker e Wigfield (1999) e Wigfield e Guthrie (1997).
Em Portugal, estudos como o de Manata (2011) e o de Menezes (2010) descrevem os perfis de leitores de diversas idades e níveis de escolaridade, utilizando como instrumentos investigativos entrevistas, questionários e inventários, muitas vezes elaborados ou adaptados pelo próprio autor da pesquisa. Esses estudos relacionam-se com a motivação para ler, pois abordam dimensões relevantes tais como frequência, preferências e hábitos de leitura, valor atribuído a essa atividade, assim como atitudes que leitores assumem quando leem em diferentes contextos e situações.

A leitura nos anos intermediários e finais da escolarização ainda é pouco investigada no Brasil (Witter, 2010) e são praticamente inexistentes os estudos que se referem à validação de instrumentos para avaliar a motivação de leitores. O mais relacionado à motivação para ler é a Escala de Atitudes de Leitura para estudantes do início do Ensino Fundamental, de Silva e Maher (1981), que foi adaptada, com a mesma finalidade, para estudantes do ensino superior por Oliveira, Santos e Oliveira (2007) e por Pellegrini, Santos e Sisto (2002), mas convém ressaltar que motivação e atitudes são constructos distintos.

\section{A Motivação para a Leitura numa Perspectiva da Teoria da Autodeterminação (TAD)}

Deci e Ryan (1985) descrevem os fundamentos da TAD, teoria sociocognitiva da motivação que explica o dinamismo do comportamento humano a partir de três necessidades básicas: autonomia, afiliação e senso de competência. Muitas das teorias tradicionais manifestam uma visão dicotômica entre a Motivação Intrínseca (MI), cujo foco é a atividade por si mesma e a Motivação Extrínseca (ME), que tem por intuito a obtenção de uma recompensa ou evitar uma punição ou atividade percebida como desagradável. A TAD, no entanto, defende um continuum motivacional constituído por Desmotivação, caracterizada pela falta de propósito e de intenção para agir, quatro formas de Motivação Extrínseca e a Motivação Intrínseca (Gillet et al., 2012; Guay et al., 2005; Guay, Vallerand, \& Blanchard, 2000).

Desta forma, a motivação individual é variável em função do nível de autonomia manifestado pela pessoa em cada contexto e situação (Vallerand \& Ratelle, 2002). Em se tratando da leitura, o continuum motivacional pode ser assim exemplificado: (a) "Não gosto de ler" (Desmotivação); (b) "Leio, na escola, para não perder nota" (ME por Regulação Externa); (c) "Leio para mostrar aos colegas que sou bom em leitura" (ME Introjetada); (d) "Leio para aprender assuntos interessantes" (ME identificada); (e) "Leio para me atualizar depois de terminar os estudos" (ME Integrada); (f) "Leio por prazer" (Motivação intrínseca). A ME por Regulação Externa e a ME Introjetada não são autodeterminadas porque a pessoa age sob pressão externa (recompensa, punição) ou interna (amor próprio, medo, culpa). Entretanto, a ME Identificada já demonstra certo grau de autonomia, pois revela a internalização de valores culturais e a ME Integrada, pressupõe que esses valores se tornam pessoais e são incorporados ao self. 
Nesses casos, o comportamento motivado e as experiências pessoais refletem maior senso de escolha, com menos conflitos internos, maior compromisso, melhor desempenho (Ratelle et al., 2007). A MI reflete autodeterminação, pois o sujeito demonstra senso de competência, de autonomia e de afiliação ao agir.

Diversos são os instrumentos para mensurar a motivação para aprender, em sintonia com essa Teoria Sociocognitiva da Motivação, tanto na literatura estrangeira (Guay et al., 2010; Guay et al., 2005; Guay et al., 2000; Vallerand et al., 1992, 1993), quanto nacional (Boruchovitch, 2008a; Guimarães \& Bzuneck, 2008; Martinelli \& Genari, 2009; Neves \& Boruchovitch, 2007; Rufini et al., 2011). Resultados provenientes de investigações com esses instrumentos confirmam a existência de todos os tipos de motivação propostos no continuum da TAD. Todavia, constata-se a ausência de escalas especificamente orientadas à avaliação da motivação para a leitura, à luz desse referencial teórico.

Tendo em vista a reconhecida relevância do constructo Motivação para a Leitura, sua importância para a aprendizagem e a escassez de instrumentos para mensurá-la em nosso meio, o presente estudo tem os seguintes objetivos: (a) descrever os passos relativos à construção de uma escala para avaliar a motivação em leitura para alunos brasileiros, à luz da Teoria da Autodeterminação; (b) apresentar a análise preliminar de suas propriedades psicométricas.

A seguir, serão descritos os passos relativos à construção e validação da Escala de Motivação para a Leitura (EML) destinada a estudantes do primeiro ciclo do Ensino Fundamental, no Brasil. Cabe mencionar que foram os estudos disponíveis na literatura acerca da motivação para aprender, tendo como referencial a TAD, que serviram de parâmetros para a presente investigação.

\section{Método}

\section{Descrição da Construção da Escala}

A redação inicial dos itens da escala buscou caracterizar comportamentos de leitores, de acordo com o continuum da Teoria da Autodeterminação, de forma a descrever a Desmotivação para a leitura, as quatro formas de ME e a MI. Para a validação do constructo e semântica dos itens, alguns procedimentos foram adotados. Em primeiro lugar foi solicitado a dois especialistas em avaliação educacional e na TAD, a sua categorização, à luz dessa teoria, tendo sido obtida uma concordância de $85 \%$ entre o pesquisador e os juízes. Em segundo lugar, foi solicitado a 20 estudantes de $5^{\circ}$ ano, que avaliassem os itens quanto à sua inteligibilidade, após o que a revisão na redação dos mesmos foi realizada e ajustes foram feitos, sempre que necessário. Dessa forma, chegou-se à versão original da Escala de Motivação para a Leitura (EML) para estudantes alfabetizados do $3^{\circ}$ ao $5^{\circ}$ anos do Ensino Fundamental, utilizada no presente estudo, e destinada ao início do Ensino Fundamental. Inicialmente foi constituída por 48 itens, dos quais seis seriam para a Desmotivação para a leitura, seis para a ME por Regulação Externa, nove para a ME Introjetada, dez para a ME Identificada, sete para a ME Integrada e dez para a Motivação Intrínseca.

$\mathrm{Na}$ folha de aplicação, após o cabeçalho, com questões referentes aos dados demográficos (gênero, idade, escola, ano de escolaridade, período) havia a seguinte proposta de instrução acerca da escala:

Gostaríamos de conhecer suas ideias sobre a leitura, o que você pensa e sente quando está lendo no seu tempo livre ou para estudar. Não existem respostas certas ou erradas. O importante é você responder com sinceridade o que realmente pensa de cada frase abaixo. Você deve ler com cuidado cada questão. Pense: O que esta frase tem a ver comigo? Você tem as seguintes opções: (3) Tem tudo a ver comigo (2) Tem alguma coisa a ver comigo (1) Tem muito pouco a ver comigo.

A seguir, foram inseridos dois exemplos, com as alternativas de respostas, para que os participantes fizessem um pequeno treino. $\mathrm{Na}$ avaliação de estudantes, "tem tudo a ver comigo", valerá três pontos, "tem alguma coisa a ver comigo" valerá dois pontos e "Tem muito pouco a ver comigo" valerá um ponto.

\section{Participantes}

Participaram desse estudo 253 estudantes do $3^{\circ}$ ao $5^{\circ}$ anos do Ensino Fundamental de duas escolas da Rede Pública Municipal de Jundiaí, Estado de São Paulo. A idade variou de sete a 11 anos; relativamente à escolarização, a amostra se distribuiu na seguinte conformidade: $18.97 \%$ frequentavam o $3^{\circ}$ ano, $42,29 \%$ encontravam-se no $4^{\circ}$ ano e $38,74 \%$, no $5^{\circ}$ ano. Quanto ao período, $81,02 \%$ estudavam pela manhã e $18,97 \%$, à tarde.

\section{Procedimentos da Coleta de Dados}

Após o projeto ter sido aprovado pelo Comitê de Ética em Pesquisa da Universidade Estadual de Campinas (UNICAMP) foi feito um contato oficial com a Secretaria de Educação do Município explicando o projeto e solicitando autorização para o acesso à pesquisa. A seguir, os dirigentes dessas duas unidades, que atendem crianças do primeiro ao quinto ano de escolaridade, foram contatados, sendo esclarecidos os objetivos da pesquisa, bem como estabelecidas as condições de parte a parte quanto aos procedimentos, horários, cronograma e outros detalhes, de modo a atender aos requisitos da pesquisa e não interferir no bom andamento das atividades escolares.

Todos os cuidados éticos foram observados e o termo de consentimento (TCLE), elaborado de acordo com as normas estabelecidas pelo Conselho Nacional de Saúde (Resolução 196/96 e suas complementares), foi distribuído para a devida autorização dos pais.

A sessão de aplicação da EML foi coletiva, para os participantes autorizados, e teve a duração aproximada de 30 minutos. Em cada turma, houve o cuidado de se estabelecer um clima de confiança e colaboração entre pesquisador e alunos, explicando-se os objetivos da pesquisa, tendo sido assegurado que as respostas não 
Gomes, M. A. M. \& Boruchovitch, E. (2015). Escala de Motivação para a Leitura para Estudantes do Ensino Fundamental: Construção e Validação.

influenciariam a avaliação escolar. Após as instruções gerais era recomendado que os estudantes levantassem a mão em caso de dúvidas e que fosse utilizada a régua para facilitar a leitura e o preenchimento das respostas. Os itens foram lidos pelo pesquisador, um a um, e dado tempo para refletirem e assinalarem o escolhido. No final era solicitado aos participantes que conferissem se todas as questões haviam sido preenchidas corretamente. Havia sempre um monitor, junto com o pesquisador, para ajudar os alunos com alguma dificuldade.

\section{Resultados e Discussão}

Os estudos para a validação da escala foram realizados por meio do programa Statistical Analysis System, versão 8.02. Para verificar a fatorabilidade da escala, efetuou-se o Teste de Esfericidade de Bartlett e o Teste de Kaiser-Meyer-Olkin (KMO). O Teste de Esfericidade de Bartlett indicou uma correlação entre os itens (X2 $=5229,82$; $g l=1128 ; p<0,001)$. A medida de adequação da amostra, averiguada pelo índice de Kaiser-Meyer-Olkin (KMO), foi de 0,87 . Os dados obtidos por esses dois procedimentos revelaram que há um nível de probabilidade muito adequado para a correlação entre variáveis e que a amostra é apropriada para a realização da Análise Fatorial. Foi então utilizado o método dos componentes principais (eigenvalue superior a 1,5). Aplicou-se, a rotação Promax em virtude de correlação encontrada entre os fatores. Para obter a matriz rotada foi estipulado o critério de carga fatorial mínima de 0,40 para a inclusão dos itens. Para avaliar a conveniência da manutenção do número de fatores estipulados, utilizou-se o Scree plot e optou-se por fixar a extração de quatro fatores (eingenvalues maior que 1,5), que explicam 42,7\% da variabilidade total, uma vez que, a partir deste fator, a curva se estabiliza, sem maiores aumentos do percentual acumulado de explicação da variância (Hatcher, 1994). Desta forma, levou-se em conta a consistência teórica e os dados fornecidos pelo gráfico de sedimentação (Figura 1). O nível de significância adotado para os testes estatísticos foi de $5 \%(p<0,05)$.

Os fatores resultantes foram então denominados: Fator 1, ME Autônoma para a Leitura, que agrupou itens que correspondem à ME Identificada e ME Integrada. O Fator 2, Desmotivação para a Leitura; o Fator 3, ME Controlada para a Leitura, incluiu itens correspondentes à ME por Regulação Externa e ME por Introjeção e, finalmente, o Fator 4, Motivação Intrínseca para a Leitura.

O Fator 1, ME Autônoma para a Leitura, foi composto por 15 itens com saturação entre 0,44 e 0,68 (itens 47,38 , 9, 44, 17, 24, 34, 37, 11, 1, 33, 6, 30, 43, 40). Apresentou eingenvalues de 10,67 , explicando $22,23 \%$ da variância total (Exemplo: "Leio para estimular a minha inteligência e o meu raciocínio"). O segundo fator, Desmotivação para a Leitura se constitui de seis itens com saturação entre 0,52 e 0,86 ; apresentou eingenvalues de 5,90 e explica $12,30 \%$ da variância total (itens $32,15,36,22,21,26$ ), como por exemplo, "Ler é muito chato". O terceiro fator,

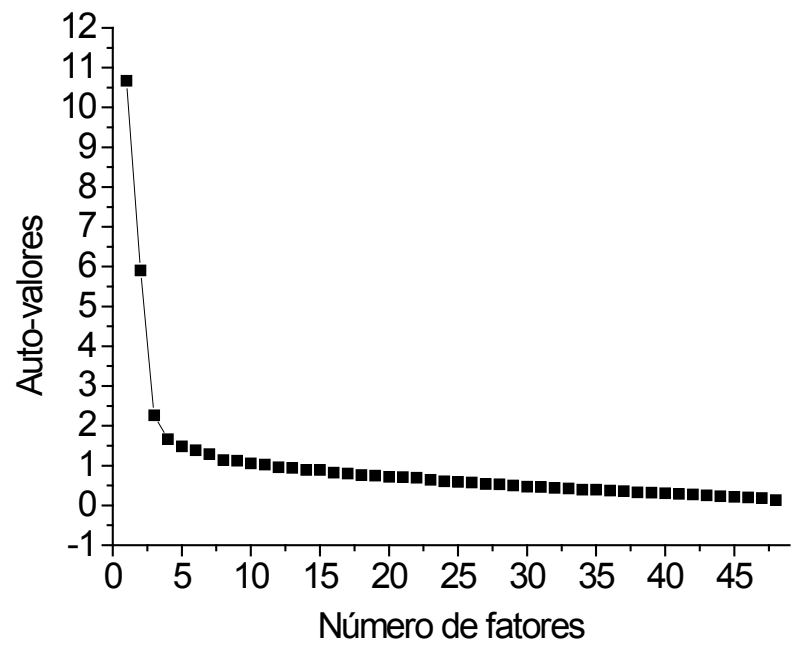

Figura 1. Teste do scree plot para número de fatores da Escala de Motivação para Leitura.

ME Controlada para a Leitura agrupou dez itens com saturação entre 0,46 e 0,74 ; apresentou eingenvalues de 2,26 , explicando $4,71 \%$ da variância total (itens 16,12 , 14, 20, 4, 5, 2, 13, 41, 29), entre os quais: "Leio somente para o professor não ficar zangado comigo". Finalmente, o quarto fator, MI para a Leitura agrupou oito itens com saturação entre 0,41 e 0,64 ; apresentou eingenvalues de 1,67 e explica $3,47 \%$ da variância total (itens $42,39,28$, $3,10,46,35,7)$, como por exemplo: "Quando eu leio não vejo o tempo passar". Outros exemplos de itens da escala podem ser encontrados no Anexo.

No processo de análise foram desconsiderados os itens 25 e 31 por terem se alocado com carga invertida no fator 2 , isto é, itens que descrevem a Motivação Intrínseca obtiveram carga invertida na Desmotivação; os itens 8, 23, 45 e 48 , por não terem obtido carga fatorial maior do que $0,40 \mathrm{em}$ nenhum dos fatores obtidos na análise fatorial exploratória. Exemplo desses itens: "Leio porque isso me permite aprender mais sobre temas (assuntos) que me interessam". Foram igualmente excluídos os itens 18,19 e 27 por terem sido considerados não coerentes com o fator onde se agregaram, como por exemplo, "Leio somente quando vale nota" caracteriza a ME por Regulação Externa e não a Desmotivação. O item 29 deveria ser descartado por obter carga alta em mais de um fator, porém foi alocado no fator de maior carga e, numa análise mais qualitativa, considerado ajustado. De fato, o item "Leio somente porque quero mostrar para mim mesmo que sou inteligente" caracteriza a ME por Introjeção e ficou no Fator 3, juntamente com outros itens da mesma categoria e com os itens de ME por Regulação Externa. Por este motivo, o fator foi denominado ME Controlada para a Leitura, da mesma forma como em Guay et al., (2000) e Ratelle et al. (2007).

Constatou-se que os 39 itens resultantes da análise fatorial se organizaram em quatro fatores revelando congruência com o continuum descrito pela TAD. De fato, na 
AMS (Vallerand et al., 1992, 1993) foram sete os fatores resultantes (três tipos de MI, três tipos de ME e a Desmotivação. Porém, na Situational Motivation Scale (SIMS), validada por Guay et al. (2000) foram extraídos quatro fatores - a Desmotivação, a MI, a ME Identificada e foram agrupadas num mesmo fator a ME por Regulação Externa e a ME Introjetada sendo esse fator, denominado Regulação Externa, estrutura semelhante à obtida no presente estudo. A Elementary School Motivation Scale (ESMS) de Guay et al., (2010; Guay et al., 2005) é constituída por apenas três desses fatores. A Desmotivação foi desconsiderada pelos autores para evitar um número excessivo de itens, uma vez que essas duas escalas anteriormente mencionadas tiveram como objetivo avaliar a motivação situacional em geral, para a leitura, a escrita e a matemática comparativamente ao autoconceito e rendimento acadêmicos nessas matérias escolares, como antecedentes e consequentes da motivação. Esses estudos, embora não relacionados especificamente à motivação para a leitura, são referência básica nos estudos da TAD, na avaliação da motivação acadêmica.

Na presente escala, o Fator 1 corresponde às formas mais autônomas de ME para a Leitura e indicam comportamentos leitores mais adaptativos, orientados por identificação ou integração de valores culturais e o Fator 4 corresponde aos itens que descrevem a MI. O Fator 2, que agregou os itens que descrevem a Desmotivação, ficou muito bem caracterizado e o Fator 3 (ME Controlada) agrupou itens relativos à ME por Regulação Externa e alguns itens relativos à ME Introjetada que, embora revele algum grau de internalização, ou seja, indícios de autocontigenciamento de estímulos e suas consequências, ainda não reflete um comportamento autodeterminado. Esses resultados são, portanto, congruentes com outros estudos realizados na perspectiva da TAD, ainda que o foco deles tenha sido a motivação acadêmica (nível contextual e situacional da motivação humana). A estrutura da escala resultante pode ser visualizada na Tabela 1 .

Tabela 1

Distribuição dos Ítens por Fator e suas Respectivas Cargas Fatoriais

\begin{tabular}{|c|c|c|c|c|c|}
\hline \multirow[b]{2}{*}{ Itens } & \multirow[b]{2}{*}{ Categorias } & \multicolumn{4}{|c|}{ Cargas Fatoriais } \\
\hline & & 1 & 2 & 3 & 4 \\
\hline EML 1 & ME Autônoma para a Leitura & 0,49 & & & \\
\hline EML 2 & ME Controlada para a Leitura & & & 0,60 & \\
\hline EML 3 & Motivação Intrínseca para a Leitura & & & & 0,56 \\
\hline EML 4 & ME Controlada para a Leitura & & & 0,69 & \\
\hline EML 5 & ME Controlada para a Leitura & & & 0,66 & \\
\hline EML 6 & ME Autônoma para a Leitura & 0,46 & & & \\
\hline EML 7 & Motivação Intrínseca para a Leitura & & & & 0,41 \\
\hline EML 8 & Não obteve carga mínima necessária & & & & \\
\hline EML 9 & ME Autônoma para a Leitura & 0,60 & & & \\
\hline EML 10 & Motivação Intrínseca para a Leitura & & & & 0,50 \\
\hline EML 11 & ME Autônoma para a Leitura & 0,52 & & & \\
\hline EML 12 & ME Controlada para a Leitura & & & 0,73 & \\
\hline EML 13 & ME Controlada para a Leitura & & & 0,54 & \\
\hline EML 14 & ME Controlada para a Leitura & & & 0,73 & \\
\hline EML 15 & Desmotivação para a Leitura & & 0,69 & & \\
\hline EML 16 & ME Controlada para a Leitura & & & 0,74 & \\
\hline EML 17 & ME Autônoma para a Leitura & 0,56 & & & \\
\hline EML 18 & Agrupamento em fator não previsto & & & & \\
\hline EML 19 & Agrupamento em fator não previsto & & & & \\
\hline EML 20 & ME Controlada para a Leitura & & & 0,71 & \\
\hline EML 21 & Desmotivação para a Leitura & & 0,57 & & \\
\hline EML 22 & Desmotivação para a Leitura & & 0,57 & & \\
\hline
\end{tabular}


Gomes, M. A. M. \& Boruchovitch, E. (2015). Escala de Motivação para a Leitura para Estudantes do Ensino Fundamental: Construção e Validação.

EML 23 Não obteve carga mínima necessária

EML 24

EML 25

EML 26

EML 27

EML 28

EML 29

EML 30

EML 31

EML 32

EML 33

EML 34

EML 35

EML 36

EML 37

EML 38

EML 39

EML 40

EML 41

EML 42

EML 43

EML 44

EML 45

EML 46

EML 47

EML 48
ME Autônoma para a Leitura

Agrupamento por inversão

Desmotivação para a Leitura

Agrupamento em fator não previsto

Motivação Intrínseca para a Leitura

ME Controlada para a Leitura

ME Autônoma para a Leitura

Agrupamento por inversão

Desmotivação para a Leitura

ME Autônoma para a Leitura

ME Autônoma para a Leitura

Motivação Intrínseca para a Leitura

Desmotivação para a Leitura

ME Autônoma para a Leitura

ME Autônoma para a Leitura

Motivação Intrínseca para a Leitura

ME Autônoma para a Leitura

ME Controlada para a Leitura

Motivação Intrínseca para a Leitura

ME Autônoma para a Leitura

ME Autônoma para a Leitura

Não obteve carga mínima necessária

Motivação Intrínseca para a Leitura

ME Autônoma para a Leitura

Não obteve carga mínima necessária

Eingenvalues

Variância explicada

Total da variância explicada
0,53

0,56

0,60

0,46

0,45

0,86

0,47

0,53

0,46

0,68

0,52

0,62

0,44

0,48

0,45

0,57

0,50

0,68

$10,67 \quad 5,90 \quad 2,26 \quad 1,67$

22,23

12,30

4,71

3,47
Embora o número de fatores identificados tenha sido quatro e não seis, como são descritos por Vallerand $\mathrm{e}$ Ratelle (2002), esses resultados são válidos, confiáveis e coerentes com a teoria da TAD e com o continuum motivacional, nos estudos em que foram apresentados de forma abreviada (Guay et al., 2010; Guay et al., 2005; Guay et al., 2000; Ratelle et al., 2007). Relativamente à precisão, a escala total $(N=39)$ alcançou um índice muito satisfatório, com coeficiente de Cronbach igual a 0,83 , bem como para todas as subescalas. $\mathrm{O}$ primeiro fator alcançou o índice de 0,87 , o segundo fator, de 0,83 , o índice do terceiro fator foi de 0,86 e o quarto fator, de 0,80 . Os resultados foram também congruentes com o modelo descrito pela TAD, isto é, as correlações entre os fatores são maiores entre as motivações adjacentes e negativas nas motivações do extremo do continuum, conforme se observa na Tabela 2.

Como se vê, a rotação oblíqua Promax mostrou-se o método mais consistente para este estudo e evidenciou, também, uma correlação positiva e significativa de 0,57 entre o fator 1 (ME Autônoma para a Leitura,) e o fator 4 (Motivação Intrínseca), ou seja, as formas de motivação mais próximas e mais autodeterminadas do continuum motivacional. Estudantes com pontuação elevada nesses itens podem ser considerados como tendo um perfil motivacional mais autônomo e denotam possuir senso de competência e autodeterminação. A correlação positiva entre esses fatores era, pois, esperada e está em consonância com a TAD, que descreve duas formas de motivação mais autodeterminadas: a ME Identificada resultante da internalização de valores culturais e a ME Integrada que 
Psicologia: Reflexão e Crítica, 28(1), 68-76.

Tabela 2

Escala de Motivação para a Leitura - Consistência Interna: Alfa de Conbach (na diagonal), Coeficientes de Correlação de Spearman (acima da diagonal)

\begin{tabular}{lcccc}
\hline $\begin{array}{l}\text { Subescalas de Motivação } \\
\text { para a Leitura }\end{array}$ & Desmotivação & ME Controlada & ME Autônoma & Motivação Intrínseca \\
\hline Desmotivação & $0,23 * * * *$ & $-0,19 * * *$ & $-0,30 * * * * *$ \\
ME Controlada & & $0,18 * *$ & $-0,03 *$ \\
ME Autônoma & & & $0,57 * * * * *$ \\
M. Intrínseca & & & & \\
$\alpha$ & 0,87 & 0,83 & 0,86 & 0,80 \\
\hline
\end{tabular}

Nota. Correlações entre os resultados da avaliação dos tipos de motivação.

${ }^{*} p=0,6593 ; * * p=0,42 ; * * * p=0,0021 ; * * * * p=0,0002 ; * * * * * p<0,0001$.

incorpora esses valores ao ego. O mesmo se pode afirmar da correlação negativa e significativa $(r=-0,30)$ entre o fator 2 (Desmotivação para a Leitura) e o fator 4 (Motivação Intrínseca para a Leitura) porque são os fatores opostos no continuum motivacional. Entre a Motivação Intrínseca e a ME Controlada para a Leitura a correlação foi nula ( $\mathrm{v}$ $=-0,03$ ). As correlações foram positivas e significativas de 0,18 entre o fator 1 (ME Autônoma para a Leitura) e o fator 3 (ME Controlada para a Leitura) e de 0,23 entre os fatores fator 2 (Desmotivação para a Leitura) e o fator 3 (ME Controlada para a Leitura), tipos de motivação adjacentes no continuum descrito pela TAD. Houve congruência entre esses resultados e a SMS de Vallerand et al. (1992, 1993), na literatura estrangeira, bem como em Guimarães e Bzuneck (2008); Rufini et al. (2011), na literatura nacional, embora esses estudos não tenham sido específicos para a leitura, mas referentes à motivação no contexto escolar, na perspectiva da TAD.

Esta Escala de Motivação para a Leitura (EML), validada para estudantes dos anos iniciais do Ensino Fundamental, além de coerente com a TAD revelou bons índices de consistência interna de cada um dos fatores (Alpha de Cronbach) e do total, entre 0,80 e 0,87, de modo semelhante aos obtidos em outros estudos (Vallerand et al., 1992, 1993). Assim, pode-se concluir que a Escala de Motivação para a Leitura (EML), construída e examinada para ser utilizada no Ensino Fundamental $\left(3^{\circ}\right.$ ao $5^{\circ}$ anos) é um instrumento válido, preciso e confiável, bem como simples e de fácil aplicação, que permite identificar as características motivacionais dos estudantes para a leitura, indicando os níveis de Motivação Intrínseca, de ME Autônoma, de ME Controlada e de Desmotivação para essa atividade.

\section{Considerações Finais}

A construção da EML sustentou-se na Teoria da Autodeterminação da Motivação e atendeu à demanda por estudos que contribuam para confirmar e ampliar os dados já existentes oriundos de pesquisas estrangeiras e nacionais.
Pretendeu identificar não apenas a Motivação Extrínseca e Intrínseca, mas avaliar a existência e a pertinência de outras formas de motivação descritas pelos pesquisadores da TAD, em amostra brasileira, especificamente em atividades de leitura, pois, de acordo com o Modelo Hierárquico da Motivação (Vallerand \& Lalande; 2011; Vallerand \& Ratelle, 2002), a motivação de cada estudante se mostra qualitativamente diferente no âmbito de cada disciplina com efeitos nos resultados escolares e em outras variáveis do contexto educacional (Guay et al., 2010; Guay et al., 2005; Guay et al., 2000; Ratelle et al., 2007).

O presente estudo, certamente, representa um passo inicial importante na tentativa de suprir a carência de instrumentos nacionais orientados à mensuração de uma variável essencial à aprendizagem que é a motivação para a leitura. Espera-se que pesquisas futuras possam avançar no sentido de superar as limitações desta investigação, por meio da realização de análises mais complexas com a escala, em amostras maiores e mais representativas. Recomenda-se que estudos com emprego da fatorial confirmatória e com base no modelo de Rasch, sejam conduzidos, de modo a se aprofundar conhecimentos para o melhor aproveitamento da escala em avaliação motivacional. Destaca-se, igualmente, a necessidade de investigações voltadas para o exame da validade preditiva e concorrente da escala, bem como da estabilidade temporal do constructo.

Esforços devem também ser direcionados no sentido da construção e validação de escalas que avaliem a motivação para a leitura de estudantes de outras faixas etárias e anos escolares, já que EML se destina apenas a estudantes das séries iniciais do Ensino Fundamental. Esses instrumentos serão úteis para que se tenha uma visão do perfil motivacional dos estudantes em leitura ao longo da Educação Básica como em Gillet et al. (2012) e Mata et al., (2009). Assim como Taboada et al. (2009) desenvolveram estudos para estabelecer correlações entre o perfil motivacional de leitores e o seu autoconceito enquanto estudantes, as escalas de motivação para a leitura validadas poderão ser utilizadas em pesquisas correlacionais que examinem as possíveis relações existentes entre a motivação para ler e constructos 
relevantes para o processo de ensino e de aprendizagem, tais como o desempenho escolar, a utilização de estratégias de aprendizagem cognitivas, metacognitivas e específicas de leitura, entre outros.

Por fim, tem-se a expectativa de que a EML para estudantes no início do Ensino Fundamental possa ser útil aos educadores interessados em diagnosticar e incrementar a motivação para a leitura entre os estudantes e ajudá-los na superação das defasagens crônicas verificadas na aprendizagem de Língua Materna no Sistema Educacional Brasileiro, desenvolvendo práticas consistentes de leitura, que, por sua vez, tenham repercussões positivas em sua aprendizagem.

\section{Referências}

Baker, L., \& Wigfield, A. (1999). Dimensions of children's motivation for reading and their relations to reading activity and reading achievement. Reading Research Quarterly, 34(4), 452-477. doi:10.1598/RRQ.34.4.4

Beker, M., McElvany, N., \& Kortenbruck, M. (2010). Intrinsic and extrinsic reading motivation as predictors of reading literacy: A longitudinal study. Journal of Educational Psychology, 102(4), 773-785. doi:10.1037/a0020084

Boruchovitch, E. (2008a). Escala de Motivação para Aprender de Universitários (EMA-U): Propriedades psicométricas. Avaliação Psicológica, 7(2), 127-134.

Boruchovitch, E. (2008b). A motivação para aprender de estudantes em cursos de formação de professores. Educação (Porto Alegre), 31(1), 30-38.

Clark, C., \& De Zoysa, S. (2011). Mapping the interrelationships of reading enjoyment, attitudes, behaviour and attainment: An exploratory investigation. National Literacy Trust.

Corpus, J. H., \& Wormington, S. V. (2011, April). Profiles of intrinsic and extrinsic motivations in elementary school. Paper presented at the annual meeting of the American Educational Research Association, New Orleans, LA.

Deci, E., \& Ryan, R. (1985). Intrinsic motivation and selfdetermination in human behavior. New York: Plenum

Gambrell, L. B., Palmer, B. M., Codling, R. M., \& Mazzoni, S. A. (1996). Assessing motivation to read. The Reading Teacher, 49(7), 1-34. doi:10.1598/RT.49.7.2

Gillet, N., Vallerand, R. J., \& Lafrenière, A. K. (2012). Intrinsic and extrinsic school motivation as a function of age: The mediating role of autonomy support. Social Psychology of Education: An International Journal, 15(1), 77-95. doi:10.1007/ s11218-011-9170-2

Gomes, M. A. M., \& Boruchovitch, E. (2011). Aprendizagem autorregulada da leitura: Resultados positivos de uma intervenção psicopedagógica. Psicologia: Teoria \& Pesquisa, 27(3), 291-299.

Guay, F., Chanal, J., Ratelle, C. F., Marsh, H. W., Larose, S., \& Boivin, M. (2010). Intrinsic identified and controlled types of motivation for school subjects in young elementary school children. British Journal of Educational Psychology, 80, 711735. doi:10.1348/000709910X499084

Guay, F., Marsh, H. W., Dowson, M., \& Larose, S. (2005, November-December). Assessing academic motivation among elementary school children: The Elementary School Motivation Scale (ESMS). Paper presented at the Australian Association for Research in Education, Conference 27, Par- ramatta, Australia. doi:10.1023/A:1005614228250

Guay, F., Vallerand, R. J., \& Blanchard, C. (2000). On the assessment of situational intrinsic and extrinsic motivation: The Situational Motivation Scale (SIMS). Motivation and Emotion, 24(3), 175-213.

Guimarães, S. E. R., \& Bzuneck, J. A. (2008). Propriedades psicométricas de um instrumento para avaliação da motivação de universitários. Ciências \& Cognição, 13, 101-113.

Guthrie, J., McRae, A., \& Klauda, S. L. (2007). Contributions of concept-oriented reading instruction to knowledge about interventions for motivations in reading. Educational Psychologist, 42(4), 237-250. doi: 10.1080/00461520701621087

Hatcher, L. (1994). A step-by-step approach to using the sas system for factor analysis and structural equation modeling. Cary, NC: Statistical Analysis System Institute.

Joly, M. C. R. A., \& Prates, E. A. R. (2011). Avaliação da Escala de Motivação Acadêmica em estudantes paulistas: Propriedades psicométricas. Psico-USF, 16(2), 175-184.

Manata, E. F. (2011). Atitudes dos jovens face à leitura e a si próprios: Um estudo com alunos do 7. ${ }^{\circ}$ e 9. ${ }^{\circ}$ ano (Dissertação de mestrado, Instituto de Educação, Universidade de Lisboa, Portugal). Recuperado em http://repositorio.ul.pt/ bitstream/10451/5684/1/ulfpie039817_tm.pdf

Martinelli, S. de C., \& Genari, C. H. M. (2009). Relações entre desempenho escolar e orientações motivacionais. Estudos de Psicologia (Natal), 14(1), 13-21.

Mata, L., Monteiro, V., \& Peixoto, F. (2009). Motivação para a leitura ao longo da escolaridade. Análise Psicológica, 4(27), 563-572.

McKenna, M. C., Kear, D. J., \& Ellsworth, R. A. (1995). Children's attitudes toward reading: A National Survey. Reading Research Quarterly, 30(4), 934-956. doi:10.2307/748205

Megliato, J. G. P. (2011). Perfis motivacionais e engajamento de adolescentes nas tarefas escolares em casa (Dissertação de mestrado, Faculdade de Educação, Universidade Estadual de Londrina, PR, Brasil). Recuperado em http://www.bibliotecadigital.uel.br/document/?code=vtls000172051

Melnick, S. A., Henk, W. A., \& Marinak, B. A. (2009). Validation of a Reader Self Perception Scale (RSPS2) for use in grades 7 and above. NERA Conference Proceedings, Paper 11. Retrieved from http://digitalcommons.uconn.edu/nera_2009/11

Menezes, I. M. S. (2010). Hábitos de leitura de alunos dos $2 .^{\circ}$ e 3. ${ }^{\circ}$ ciclos do ensino básico e impacto na aprendizagem. Concepções de alunos, professores e professores_bibliotecários (Dissertação de mestrado em Gestão da Informação e Bibliotecas Escolares, Universidade Aberta de Lisboa, Portugal). Recuperado em http://repositorioaberto.univ-ab. pt/handle/10400.2/1678

Monteiro, V., Mata, L., \& Peixoto, F. (2008, out.). Escala de autopercepção de leitor. In A. P. Noronha, C. Machado, L. Almeida, M. Gonçalves, S. Martins, \& V. Ramalho (Eds.), Actas da XIII Conferência Internacional de Avaliação Psicológica: Formas e Contextos. Braga, Portugal: Universidade do Minho.

Neves, E. R. C., \& Boruchovitch, E. (2007). Escala de Avaliação da Motivação para Aprender de alunos do Ensino Fundamental (EMA). Psicologia: Reflexão e Crítica, 20(3), 406-413.

Oliveira, K. L., Boruchovitch, E., \& Santos, A. A. A. (2008). Leitura e desempenho escolar em português e matemática no ensino fundamental. Paidéia (Ribeirão Preto), 18(41), 531-540.

Oliveira, K. L., Santos, A. A. A., \& Oliveira, E. Z. (2007). Estudio de las propiedades psicométricas de una escala de actitudes de lectura para universitarios. Paradigma, 28(2), 165-180. 
Organisation de Coopération et de Développement Économiques. (2011). Résultats du PISA 2009: tendances dans l'apprentissage : l'évolution de la performance des élèves depuis 2000 (Vol. 5). Paris: Author.

Pellegrini, M. C. K., Santos, A. A. A., \& Sisto, F. F. (2002). Evaluación de lás actitudes de lectura en universitarios. Lectura y Vida, 23(2), 26-33.

Prates, E. A. R., Joly, M. C. R. A., Dias, A. S., \& Almeida, L. S. (2011, nov.). Competências de estudo e motivação para a universidade. Trabalho apresentado no VIII Congresso Iberoamericano de Avaliação/Evaluación Psicológica, XV Conferência Internacional Avaliação Psicológica: Formas e Contextos. Lisboa, Portugal: Sociedade Portuguesa de Psicologia.

Ratelle, C. F., Guay, F., Vallerand, R. J., Larose, S., \& Senécal, C. (2007). Autonomous, controlled, and amotivated types of academic motivation: A person-oriented analysis. Journal of Educational Psychology, 99(4), 734-746. doi:10.1037/00220663.99.4.734

Rosecler, A., \& Guimarães, S. E. R. (2010). Orientações motivacionais de alunos do curso de biblioteconomia. Revista Semestral da Associação Brasileira de Psicologia Escolar e Educacional, 14(2), 211-220.

Rufini, S. E., Bzuneck, J. A., \& Oliveira, K. L. (2011). Estudo de validação de uma medida de avaliação da motivação para alunos do ensino Fundamental. Psico-USF, 16(1), 1-9.

Silva, E. T., \& Maher, J. P. (1981). Questionário para avaliar atitudes de leitura de alunos de $1^{\circ}$ e $2^{\circ}$ Graus. In Resumos do $3^{\circ}$ Congresso de Leitura do Brasil (pp. 19-26). Campinas, SP: Universidade Estadual de Campinas.

Taboada, A., Tonks, S. M., Wigfield, A., \& Guthrie, J. T. (2009). Effects of motivational and cognitive variables on reading comprehension. Reading and Writing: An Interdisciplinary Journal, 22, 85-106. doi:10.1007/s11145-008-9133-y

Vallerand, R. J., \& Lalande, D. R. (2011). The MPIC Model: The perspective of the hierarchical model of intrinsic and extrinsic motivation. Psychological Inquiry: An International Journal for the Advancement of Psychological Theory, 22(1), 45-51. doi:10.1080/1047840X.2011.545366
Vallerand, R. J., Pelletier, L. G., Blais, M. R., Briere, N. M., Senécal, C., \& Vallières, E. (1992). The Academic Motivation Scale: A measure of intrinsic, extrinsic, and amotivation in education. Educational and Psychological Measurement, 52, 1003-1017. doi:10.1177/0013164492052004025

Vallerand, R. J., Pelletier, L. G., Blais, M. R., Brière, N. M., Senécal, C., \& Vallières, E. F. (1993). On the assessment of intrinsic, extrinsic, and amotivation in education: Evidence on the concurrent and construct validity of the Academic Motivation Scale. Educational and Psychological Measurement, 53, 159-172. doi:10.1177/0013164493053001018

Vallerand, R. J., \& Ratelle, C. F. (2002). Intrinsec and extrinsec motivation: A hierarchical model. In E. L. Deci \& R. M. Ryan, Handbook of self-determination research (pp. 37-66). New York: University of Rochester Press.

Wigfield, A., \& Guthrie, J. T. (1997). Relations of children's motivation for reading to the amount and breadth of their reading. Journal of Educational Psychology, 89, 420-432. doi:10.1037/0022-0663.89.3.420

Witter, G. P. (2010). Motivação e leitura. In: E. Boruchovitch, J. A. Bzuneck, \& S. E. R. Guimarães, Motivação para aprender - Aplicações no contexto educativo (pp. 169-192). Petrópolis, RJ: Vozes.

Wormington, S. V., Corpus, J. H., \& Anderson, K. G. (2011, April). A person centered investigation of academic motivation performance and engagement in a high school setting. Paper presented at the annual meeting of the American Educational Research Association, New Orleans, LA.

Zenorini, R. da P. C., \& Santos, A. A. A. (2010). Escala de Metas de Realização como Medida da Motivação para Aprendizagem. Revista Interamericana de Psicología / Interamerican Journal of Psychology, 44(2), 291-298.

Zenorini, R. da P. C., Santos, A. A. A., \& Monteiro, R. de M. (2011). Motivação para aprender: Relação com o desempenho de estudantes. Paidéia (Ribeirão Preto), 21(49), 157-164.

Recebido: 13/11/2013

Aceite final: 05/12/2013

Anexo

\section{Exemplos de Itens da EML para Estudantes do $3^{\circ}$ ao $5^{\circ}$ anos do Ensino Fundamental}

\section{ME Autônoma para a Leitura}

EML47 - Leio porque isso é importante para ser alguém na vida.

EML9 - Leio porque sei que a leitura é importante para o meu futuro.

Desmotivação para a Leitura

EML32 - Não tenho vontade de ler.

EML15 - Não gosto de ler.

ME Controlada para a Leitura

EML16 - Leio somente porque meus pais acham importante.

EML14 - Leio na classe apenas porque não quero que me chamem de burro.

Motivação Intrínseca para a Leitura

EML42 - Leio para relaxar.

EML28 - Quando eu leio não vejo o tempo passar. 